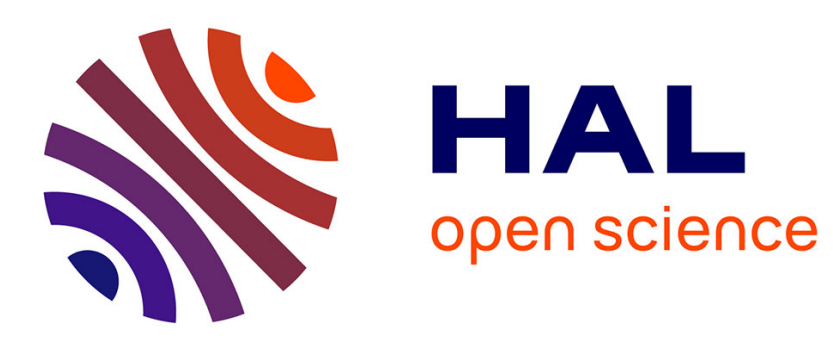

\title{
Single-file diffusion of particles with long-range interactions: Damping and finite-size effects
} Jean-Baptiste Delfau, Christophe Coste, Michel Saint-Jean

\section{To cite this version:}

Jean-Baptiste Delfau, Christophe Coste, Michel Saint-Jean. Single-file diffusion of particles with long-range interactions: Damping and finite-size effects. Physical Review E , 2011, 10.1103/PhysRevE.84.011101 . hal-01404847

\section{HAL Id: hal-01404847 \\ https://hal-univ-paris.archives-ouvertes.fr/hal-01404847}

Submitted on 29 Nov 2016

HAL is a multi-disciplinary open access archive for the deposit and dissemination of scientific research documents, whether they are published or not. The documents may come from teaching and research institutions in France or abroad, or from public or private research centers.
L'archive ouverte pluridisciplinaire HAL, est destinée au dépôt et à la diffusion de documents scientifiques de niveau recherche, publiés ou non, émanant des établissements d'enseignement et de recherche français ou étrangers, des laboratoires publics ou privés. 


\title{
Single-file diffusion of particles with long-range interactions: Damping and finite-size effects
}

\author{
Jean-Baptiste Delfau, Christophe Coste, and Michel Saint Jean \\ Laboratoire Matiere et Systemes Complexes, UMR CNRS 7057 et Université Paris Diderot-Paris 7, Bâtiment Condorcet, \\ 10 rue Alice Domon et Léonie Duquet, 75205 Paris Cedex 13, France
}

(Received 18 March 2011; published 5 July 2011)

\begin{abstract}
We study the single file diffusion of a cyclic chain of particles that cannot cross each other, in a thermal bath, with long-ranged interactions and arbitrary damping. We present simulations that exhibit new behaviors specifically associated with systems of small numbers of particles and with small damping. In order to understand those results, we present an original analysis based on the decomposition of the particles' motion in the normal modes of the chain. Our model explains all dynamic regimes observed in our simulations and provides convincing estimates of the crossover times between those regimes.
\end{abstract}

DOI: 10.1103/PhysRevE.84.011101

PACS number(s): 05.40.-a, 66.10.cg, 47.57.eb

\section{INTRODUCTION}

When Brownian particles are confined along a line in a quasi-one-dimensional channel so narrow that they cannot cross each other, anomalous diffusion appears and strongly subdiffusive behavior can be observed. This phenomenon called single-file diffusion (SFD) was first noticed in 1955 by Hodgkin and Keynes [1] who were studying water transport through molecular-sized channels in biological membranes. Since then, SFD also appeared in the diffusion of molecules in porous materials like zeolites [2-5], of charges along polymer chains [6], of ions in electrostatic traps [7], of vortices in band superconductors [8,9], and of colloids in nanosized structures [10-15] or optically generated channels $[16,17]$. Even though SFD can be encountered in a lot of various physical systems, most of the theoretical studies devoted to it are generally restricted to the simplest case: an infinite overdamped system with hard core interactions.

In this paper, we present simulation results concerning finite systems of long-range interacting particles. In particular, we focus on the dependency of the diffusion properties with the number of particles $N$ and the damping coefficient $\gamma$. We exhibit new behaviors, specifically associated with systems of small numbers of particles and with small damping. In order to interpret those results, we present an original analysis based on the decomposition of the particles' motion in the normal modes of the chain.

In the thermodynamic limit (infinite systems with finite density $\rho$ ), for overdamped dynamics with hard core interactions, several analytical models [18-22] predict that at long times, the mean square displacement [MSD; see below Eq. (13)] of a particle of mass $m$ grows as $F_{H} \sqrt{t}$ with the mobility $F_{H}$ given by

$$
F_{H}=\frac{2}{\rho} \sqrt{\frac{D_{0}}{\pi}}=\frac{2}{\rho} \sqrt{\frac{k_{B} T}{\pi m \gamma}}
$$

with $D_{0}=k_{B} T /(m \gamma)$ being the single-particle free diffusion coefficient at temperature $T$ and $k_{B}$ being Boltzmann's constant. If the interactions are long ranged, only two analytical approaches have been undertaken so far [23,24], for overdamped systems in the thermodynamic limit. There it is proven that the MSD grows as $F_{S} \sqrt{t}$ at long times, with a mobility $F_{S}$ that depends on the interaction potential, through the isothermal compressibility $\kappa_{T}$ [25] or the spring constant $K \equiv \rho / \kappa_{T}$,

$$
F_{S}=\frac{2}{\rho} S(0,0) \sqrt{\frac{D_{\mathrm{eff}}}{\pi}}=2 k_{B} T \sqrt{\frac{\kappa_{T}}{\pi m \gamma \rho}}=2 k_{B} T \frac{1}{\sqrt{\pi m \gamma K}},
$$

where $S(0,0) \equiv S(q \rightarrow 0, t=0)$ is the long-wavelength static structure factor of the particles. The diffusivity $D_{\text {eff }}$ is the effective diffusivity of a Brownian particle, taking into account its interactions with the other Brownian particles [26], and differs from the single-particle diffusivity $D_{0}$. In its last version, the expression (2) can be interpreted by considering that we can derive $F_{S}$ from $F_{H}$ if we replace the interparticle distance $1 / \rho$ by the mean square displacement $k_{B} T / K$ of a particle in the potential well due to its neighbors [25]. In Appendix B, we recover the formula (2) without the assumption of overdamped Langevin dynamics (3).

In numerical simulations and experiments, the systems are obviously finite. Periodic boundary conditions are used in simulations, and annular geometries in experiments. As a consequence of finite-size effects, the asymptotic behavior at long time for the MSD is always $D_{N} t$. All particles in the system are then totally correlated and diffuse as a single effective particle of mass $N m$ [20]. We recover it from our analytical model and provide measurements of the diffusion coefficient $D_{N}$ in good agreement with this interpretation. The SFD regime may nevertheless be observed in finite systems if the damping and the particles number are high enough, in a manner that will be clarified by our approach of finite systems dynamics.

However, most theoretical and experimental studies have been performed for overdamped systems only. This is the initial assumption in the existing models for long-range interacting particles $[23,24]$. The relevant experiments were generally done with solutions of colloids [10-17] for which overdamping is a safe assumption. The simulations [27,28] are shown in Ref. [25] to be in good agreement with the theoretical prediction of Kollmann, but they also assume overdamping in the choice of the simulation algorithm. In order to explore underdamped systems, we have previously studied the diffusion in a circular channel of millimetric steel balls electrically charged [25]. In this experiment, identical metallic beads are held in a plane horizontal condenser made 


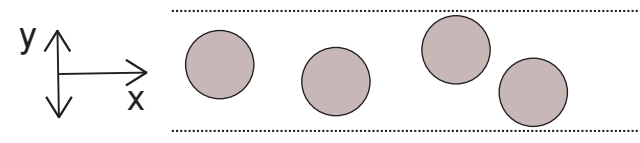

FIG. 1. (Color online) Scheme of the system.

of a silicon wafer and a glass plate covered with an optically transparent metallic layer. A constant voltage is applied to the electrodes, inducing a charge distribution of the beads. The condenser is fixed on loudspeakers excited with a white noise voltage, and we have checked that this mechanical shaking behaves as an effective thermal bath $[25,29,30]$. In this system the measurement of the damping constant $\gamma$ proved that the balls' diffusion is underdamped [29]. We have observed that the MSD of the particles exhibit the SFD scaling predicted for overdamped systems, with a prefactor that is only slightly higher than the theoretical prediction (2). Unfortunately, we were not able to tune the damping constant experimentally. Thus, in order to investigate the specific role of damping on the diffusion of finite systems we have developed numerical simulations that allow easy changes of the damping constant.

The paper is organized as follows. Section II is devoted to the description of the algorithm used in our numerical simulation. In Sec. III, we present our numerical results and exhibit new behaviors specific to systems of small numbers of underdamped particles. We characterize the various regimes for the MSD scaling with time and define the crossover times between those regimes. In Sec. IV, we give a physical interpretation of those regimes in the framework of our analytical model. We recover the various scaling laws for the MSD and provide estimates of the various crossover times, showing their dependency on the damping and particle numbers. We summarize our results in Sec. V. Two appendices are devoted to complementary calculations.

\section{DESCRIPTION OF THE SIMULATION}

\section{A. A line of particles with long-ranged interactions}

We consider point particles of mass $m$ located in the $x y$ plane, submitted to a thermal bath at temperature $T$. The particles are confined by a quadratic potential in $y$ in such a way that they cannot cross each other, as if they were diffusing in a narrow channel (see Fig. 1). This lateral confinement is chosen to mimic as well as possible experimental situations. We have checked that its strength does not influence the system behavior provided the beads stay ordered.

We describe the dynamics with the Langevin equation. Let $\mathbf{r}_{i}=\left(x_{i}, y_{i}\right)$ be the position of the particle $i$. We do not take into account the gravity, thus describing horizontal systems. The particle is submitted to a confinement force $-\beta y_{i} \mathbf{e}_{y}$ of stiffness $\beta$ and to the interaction potential $U\left(\mathbf{r}_{i}\right)$, so that the Langevin equation reads

$$
\ddot{\mathbf{r}}_{i}+\gamma \dot{\mathbf{r}}_{i}+\frac{\nabla U\left(\mathbf{r}_{i}\right)}{m}+\frac{\beta}{m} y_{i} \mathbf{e}_{y}=\frac{\boldsymbol{\mu}(t)}{m}
$$

with $\gamma$ the damping constant and $\boldsymbol{\mu}$ a random force. In our simulations, the random force has the statistical properties of a white Gaussian noise. Therefore, its components on both axes must satisfy

$$
\begin{gathered}
\left\langle\mu_{x}(t)\right\rangle=0, \quad\left\langle\mu_{y}(t)\right\rangle=0, \quad\left\langle\mu_{x}(t) \mu_{y}\left(t^{\prime}\right)\right\rangle=0, \\
\left\langle\mu_{x}(t) \mu_{x}\left(t^{\prime}\right)\right\rangle=\left\langle\mu_{y}(t) \mu_{y}\left(t^{\prime}\right)\right\rangle=2 k_{B} T m \gamma \delta\left(t-t^{\prime}\right),
\end{gathered}
$$

where $\langle\cdot\rangle$ means statistical averaging.

It is suitable to put those equations in dimensionless form, defining the following dimensionless variables: $t=\tilde{t} / \gamma$ and $x=\tilde{x} \sqrt{k_{B} T /\left(m \gamma^{2}\right)}$. It gives us

$$
\ddot{\overrightarrow{\mathbf{r}}_{i}}+\dot{\mathbf{r}_{i}}+\widetilde{\nabla} \tilde{U}\left(\widetilde{\mathbf{r}_{i}}\right)+\frac{\beta}{m \gamma^{2}} \tilde{y_{i}} \mathbf{e}_{y}=\tilde{\boldsymbol{\mu}}(\widetilde{t}),
$$

with the dimensionless quantities

$$
\widetilde{U}\left(\widetilde{\mathbf{r}_{i}}\right)=\frac{U\left(\widetilde{\mathbf{r}_{i}}\right)}{k_{B} T}, \quad \tilde{\boldsymbol{\mu}}(\widetilde{t})=\frac{\boldsymbol{\mu}(\widetilde{t})}{\sqrt{k_{B} T m \gamma^{2}}},
$$

and the only nonzero correlation (5) now reads

$$
\left\langle\tilde{\mu}_{x}(\widetilde{t}) \tilde{\mu}_{x}\left(\tilde{t}^{\prime}\right)\right\rangle=\left\langle\tilde{\mu}_{y}(\widetilde{t}) \tilde{\mu}_{y}\left(\tilde{t}^{\prime}\right)\right\rangle=2 \delta\left(\widetilde{t}-\tilde{t}^{\prime}\right) .
$$

For the sake of simplicity, we drop the "tildes" $(\sim)$ in the rest of this section.

In order to allow a direct comparison between simulations and experiments, we take the same interaction potential as in our experimental setup [25,29,30]. It reads

$$
U\left(\mathbf{r}_{i}\right)=U_{0} \sum_{j \neq i} K_{0}\left(\frac{\left|\mathbf{r}_{i}-\mathbf{r}_{j}\right|}{\lambda}\right),
$$

where $K_{0}$ is the modified Bessel function of second order and index 0 , and $\lambda$ and $U_{0}$ are two constants. In principle, the sum extends to all particles, but in practice the summation is limited to the first five neighbors of each particle, which ensures a relative precision better than $10^{-7}$ and reduces the calculation time.

To decrease the computation time further, we replace the Bessel functions in the expression of the force $\mathbf{F}\left(\mathbf{r}_{i}\right)=-\nabla U\left(\mathbf{r}_{i}\right)=\sum_{j \neq i} F_{i j}\left(\left|\mathbf{r}_{i}-\mathbf{r}_{j}\right|\right) \frac{\mathbf{r}_{i}-\mathbf{r}_{j}}{\left|\mathbf{r}_{i}-\mathbf{r}_{j}\right|}$ by asymptotic expressions,

$$
\begin{cases}F_{i j}(x)=\frac{U_{0}}{\lambda}\left[-\frac{1}{x}+b x+c x \ln (x)\right] & \text { for } x \leqslant 1, \\ F_{i j}(x)=\frac{U_{0}}{\lambda}\left[\sqrt{\frac{\pi}{2 x}} e^{-x}\left(1+\frac{a}{x}\right)\right] & \text { for } x \geqslant 1,\end{cases}
$$

where $a, b$, and $c$ are constants. They are chosen in such a way that the force and its derivative are continuous for $x=1$ and that the force is equal to its actual value at this point. Those two approximations fit very well the actual force (see Fig. 2).

\section{B. Algorithm}

The simulation is based on the Gillespie algorithm [31,32] that allows a consistent time discretization of the Langevin equation (6). We introduce a time step value $\Delta t$, which for consistency has to be much smaller than any other characteristic time scale of the system. In dimensionless units $\Delta t=10^{-3}$. Then the velocities $\dot{x}_{i}(t+\Delta t)$ and $\dot{y}_{i}(t+\Delta t)$ are calculated from updating the formula derived from Eq. (6), 


$$
\left\{\begin{array}{l}
\dot{x}_{i}(t+\Delta t)=\dot{x}_{i}(t)-\left[\dot{x}_{i}(t)+\nabla U\left(r_{i}(t)\right) \cdot \mathbf{e}_{x}\right] \Delta t+\sqrt{2 \Delta t} \mu_{x}(t), \\
\dot{y}_{i}(t+\Delta t)=\dot{y}_{i}(t)-\left[\dot{y}_{i}(t)+\frac{\beta}{m \gamma^{2}} y_{i}(t)+\nabla U\left(r_{i}(t)\right) \cdot \mathbf{e}_{y}\right] \Delta t+\sqrt{2 \Delta t} \mu_{y}(t),
\end{array}\right.
$$

where $r_{i}(t)=\sqrt{x_{i}(t)^{2}+y_{i}(t)^{2}}$. The positions $x_{i}(t+\Delta t)$ and $y_{i}(t+\Delta t)$ of all the particles are then calculated from

$$
\begin{aligned}
& x_{i}(t+\Delta t)=x_{i}(t)+\dot{x}_{i}(t) \Delta t, \\
& y_{i}(t+\Delta t)=y_{i}(t)+\dot{y}_{i}(t) \Delta t .
\end{aligned}
$$

The components of the random noise $\mu_{y}$ and $\mu_{x}$ are sampled in such a way that they have the properties given by Eqs. (4) and (8); hence they are unit normal random numbers.

We simulate systems of $N$ particles, with periodic boundary conditions. We get from Eq. (12) $N$ equivalent trajectories, because all beads play the same role. The system is simulated during a dimensionless time of $10^{3}$, which means $10^{6}$ time steps. The quantity of interest is the MSD along the $x$ direction,

$$
\left\langle\Delta x^{2}(t)\right\rangle=\left\langle\left[x\left(t+t_{0}\right)-x\left(t_{0}\right)-\left\langle x\left(t+t_{0}\right)-x\left(t_{0}\right)\right\rangle\right]^{2}\right\rangle,
$$

where $t_{0}$ is an arbitrary initial time. The ensemble averaging is done on every bead, since they all play an equivalent role. Moreover, the phenomenon is assumed to be stationary, so that $\Delta x^{2}(t)$ do not depend on $t_{0}$. For a given time $t$, it thus makes sense to average on the initial time $t_{0}$. Let $n$ be the overall number of time steps in one simulation, and $n_{t}=t / \Delta t$. Then the averaging on the initial time $t_{0}$ reads

$$
\begin{aligned}
\left\langle\left\langle\Delta x^{2}(t)\right\rangle_{e}\right\rangle_{0}= & \sum_{i=0}^{n-n_{t}} \frac{\left\{x\left[\left(n_{t}+i\right) \Delta t\right]-x(i \Delta t)\right\}^{2}}{n-n_{t}+1} \\
& -\left(\sum_{i=0}^{n-n_{t}} \frac{x\left[\left(n_{t}+i\right) \Delta t\right]-x(i \Delta t)}{n-n_{t}+1}\right)^{2},
\end{aligned}
$$

where the index $i$ is such that $t_{0}=i \Delta t,\langle\cdot\rangle_{e}$ means ensemble averaging, and $\langle\cdot\rangle_{0}$ means averaging on the initial time $t_{0}$. This way of averaging greatly improves the statistics when $n_{t}$ is smaller than $n$. We use it henceforward, denoting it with the simplified notation $\langle\cdot\rangle$ except in Appendix A where it is specifically discussed.

\section{Orders of magnitude of the various parameters}

In our simulations, we work at densities $\rho=33,100$, and 533 particles per meter. The temperature and interaction strength are such that $\Gamma$ ranges as in the experiments in Refs. [10,16,17,25] and the numerical simulations in Refs. $[27,28,33]$. The interest of the simulations is to get access to parameter values that are difficult or impossible to obtain experimentally. We vary the particle number $N$ between 32 and 1024. This last value is comparable to some simulations $[27,28]$ but much greater than in experiments [10,16,17,25]. We vary the damping constant $\gamma$ between 0.1 and $60 \mathrm{~s}^{-1}$, extending the experimental range toward small values of $\gamma$. This is to be compared to the cutoff frequency of the chain (see Sec. IV A). With our damping constant range, we get access to both the overdamped and the underdamped dynamics of the particles and are thus able to exhibit the subtle behaviors linked to underdamping.

We simulate the same system that was experimentally studied in Ref. [25]. In the experiments, the bead number $N$ varies between 12 and 37 , and the density $\rho$ is 477,566 , or 654 particles per meter. The mean interparticle distance is thus such that $1.53<1 / \rho<2.10 \mathrm{~mm}$, to be compared to the range $\lambda=0.48 \mathrm{~mm}$ of the potential. The dimensionless potential energy is such that $6<\Gamma<55$. The damping constant $\gamma$ ranges between 10 and $30 \mathrm{~s}^{-1}$ (see Ref. [29], Fig. 6). For the experimental values of density and potential energy, the cutoff frequency ranges between 21 and $37 \mathrm{~s}^{-1}$. Experimentally, we are thus in the underdamped regime, as was already noticed in Ref. [25].

\section{SFD OF FINITE SYSTEMS: THE DIFFERENT REGIMES}

In this section, we present our results about the evolution of the MSD $\left\langle\Delta x^{2}(t)\right\rangle$ as a function of the time $t$ and focus on the effects of the particle number $N$ (at fixed density) and on the damping constant $\gamma$. Two typical examples are provided by Fig. 4. The evolution of the MSD may be described by the power law $\left\langle\Delta x^{2}(t)\right\rangle \propto t^{\alpha}$, with an exponent $\alpha$ that depends on the observation time. The interpretation detailed in Sec. IV allows us to regroup them into three different regimes:

(i) During regime I, $0 \leqslant t \leqslant \tau_{\text {ball }}$, the MSD grows according to $H_{1} t^{2}$.

(ii) during regime II, $\tau_{\text {ball }} \leqslant t \leqslant \tau_{\text {coll }},\left\langle\Delta x^{2}\right\rangle$ may be proportional to $D t$ only, to $F_{S} \sqrt{t}$ only, or to $D t$ and then $F_{S} \sqrt{t}$, depending on the parameters of the simulation. The coefficient $D$ is not necessarily the free diffusion constant $D_{0}$. When both

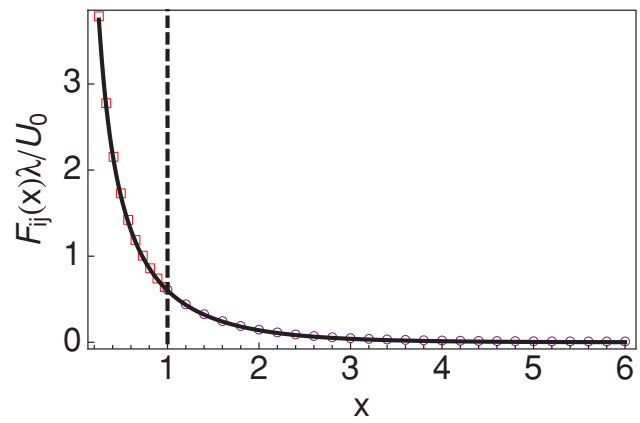

FIG. 2. (Color online) Force approximation. The thick black line represents the actual force derived from Eq. (9), the open squares and open circles are, respectively, the logarithmic and exponential approximations in Eq. (10). The two approximations are matched at $x=1$. 

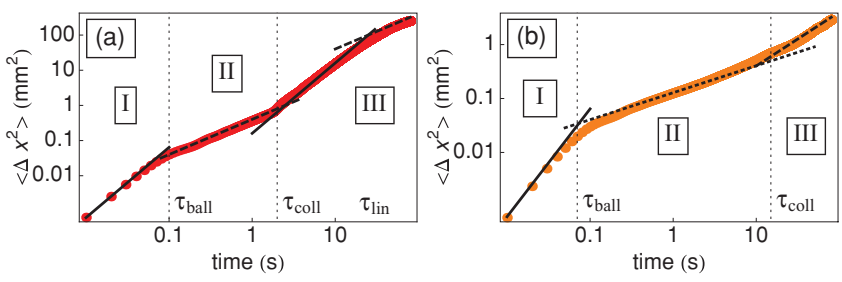

FIG. 3. (Color online) Evolution of the MSD (in $\mathrm{mm}^{2}$ ) according to the time (in s) for a chain of 32 particles with density $533 \mathrm{~m}^{-1}$, temperature $T=10^{12} \mathrm{~K}$, and interaction potential $\Gamma \approx 7$. The solid line scales as $t^{2}$, the dashed line scales as $t$, and the dotted line scales as $t^{1 / 2}$. (a) Damping constant $\gamma=0.1 \mathrm{~s}^{-1}$. In this low damping case, regime II is characterized by a $t$ scaling and regime III by a $t^{2}$ then a $t$ scaling. (b) Damping constant $\gamma=60 \mathrm{~s}^{-1}$. In this strong damping case, regime II is characterized by a $\sqrt{t}$ scaling and regime III by a $t$ scaling.

scalings $D t$ and $F_{S} \sqrt{t}$ are observed, we define the crossover time $\tau_{\text {sub }}$ between them.

(iii) A final regime, regime III, takes place for $\tau_{\text {coll }} \leqslant t$, $\left\langle\Delta x^{2}\right\rangle=D_{N} t$ at long times with $D_{N} \neq D$ and $D_{N} \neq D_{0}$. This final asymptotic behavior is sometimes preceded by the scaling $H_{N} t^{2}$ with $H_{N} \neq H_{1}$, the crossover time being denoted as $\tau_{\text {lin }}$.

\section{A. The small-time regime (regime I)}

Regime I is defined by an evolution, $\left\langle\Delta x^{2}\right\rangle=H_{1} t^{2}$. It is observed in all data displayed in Fig. 4 , and the prefactor $H_{1}$ is independent of the damping constant [see Fig. 4(a)], of the system size [see Fig. 4(b)], and of the interaction potential $\Gamma$
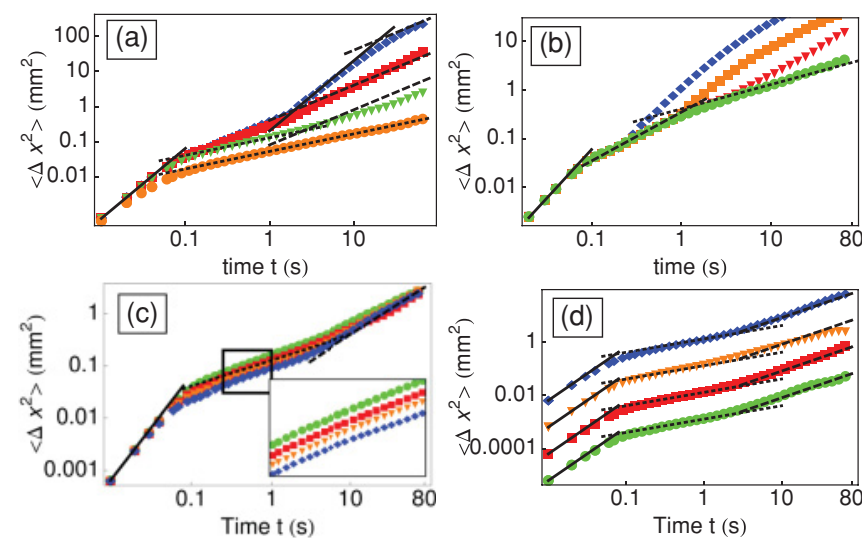

FIG. 4. (Color online) Plot of $\left\langle\Delta x(t)^{2}\right\rangle$ (in $\mathrm{mm}^{2}$ ) according to the time (in s) for a density $\rho=533 \mathrm{~m}^{-1}$. Unless otherwise specified, the parameters are $N=32, T=10^{12} \mathrm{~K}, \Gamma \approx 6.8$, and $\gamma=10 \mathrm{~s}^{-1}$. Specific values are as follows: (a) $\gamma=0.1,1,10$, and $60 \mathrm{~s}^{-1}$ (blue diamonds, red squares, green triangles, and orange disks, respectively). (b) $\gamma=1 \mathrm{~s}^{-1}$ and $N=4,16,64$, and 128 (blue diamonds, orange squares, red triangles, and green disks, respectively). (c) $\Gamma \approx$ 4.4, 6.8, 9.8, and 13.4 (green disks, red squares, orange triangles, and blue diamonds, respectively). (d) $T=10^{10}, 10^{11}, 10^{12}$, and $10^{13}$ $\mathrm{K}$, (green disks, red squares, orange triangles, and blue diamonds, respectively). The black thick line is Eq. (35), the dashed line is $F_{S} \sqrt{t}$ with the mobility $F_{S}$ given by Eq. (2), and the dotted line is either $D_{N} t$ with $D_{N}$ given by Eq. (18), in (a), (c), and (d) or $D t$ with $D$ given by Eq. (16) in (b). There are no free parameters in the calculations. [see Fig. 4(c)]. In this time range $\left(0 \leqslant t \leqslant \tau_{\text {ball }}\right)$, each particle behaves independently of the others and ensures a ballistic flight at its thermal velocity $\sqrt{k_{B} T / m}$, so that the constant $H_{1}$ should thus be equal to $k_{B} T / m$. The duration of this first ballistic regime is called $\tau_{\text {ball }}$. From our data summarized in Fig. 4, we measure the constant $H_{1}$ and show in Fig. 5(a) that it is indeed in perfect agreement with its predicted value.

This behavior is obviously not observed in the simulations of the overdamped Langevin equation [15,27,28,34], but has already been seen in simulations of the full dynamics $[35,36]$.

\section{B. The intermediate time regime (regime II)}

If we consider now the second regime, two different behaviors with distinct power laws can be observed: Fig. 4(a) shows that for the highest values of $\gamma,\left\langle\Delta x^{2}\right\rangle$ only grows as $\sqrt{t}$. When $\gamma$ is decreased, a linear evolution in $D t$ appears for $\tau_{\text {ball }}<t<\tau_{\text {sub }}$. For the lowest values of $\gamma=1 \mathrm{~s}^{-1}$ and $\gamma=0.1 \mathrm{~s}^{-1}$, the $\sqrt{t}$ scaling completely disappears. This is a finite-size effect, as demonstrated by Fig. 4(b). The data displayed in this picture are recorded at a low value, $\gamma=1$ $\mathrm{s}^{-1}$, and the $\sqrt{t}$ scaling is indeed recovered at large numbers of particles, typically $N>128$. Data from simulations with 256, 512, and 1024 particles (at constant density) superimpose exactly on the data for 128 particles.

We could be tempted to explain the linear evolution in $D t$ by arguing that we observe the diffusion of a free particle that needs a finite time to feel the effect of confinement. If this should be the case, the diffusion coefficient $D$ should be the diffusion constant for a free particle, which is

$$
D_{0}=\frac{k_{B} T}{m \gamma} \text {. }
$$

In Fig. 5(b), we compare our numerical values of $D$ to $D_{0}$. It is obvious that $D$ is very different from $D_{0}$ except at the lowest values of the density $\rho$ (that is, low interactions). We see in Sec. IV D that for high interactions the coefficient $D$ actually results from a collective behavior of the particles. In our model [see Eq. (42)], when $\rho$ is high (high interactions), the coefficient $D$ doesn't depend upon $\gamma$ and is given by

$$
D=\frac{k_{B} T}{2 \pi} \sqrt{\frac{\kappa_{T}}{m \rho}}=\frac{k_{B} T}{2 \pi \sqrt{m K}} .
$$

In Fig. 5(c), we see that at high density the coefficient $D$ is actually a function of $k_{B} T / \sqrt{m K}$, but with a numerical coefficient that is rather equal to $1 / 2$. The dependency of $D$ on either the spring constant $K=U^{\prime \prime}(1 / \rho)$ or the compressibility $\kappa_{T}=\rho / K$ indicates that collective phenomena are responsible of this behavior and that the "free particle" hypothesis does not account for the linear behavior observed in strongly interacting systems. The modified Bessel function $K_{0}$, which gives the behavior of the potential $U(1 / \rho)$ [see (9)], is a very quickly increasing function of the density, which explains why this behavior is typical of high density systems.

When the subdiffusive regime $\left\langle\Delta x^{2}\right\rangle=F \sqrt{t}$ is observed, as in Figs. 4(c) and Fig. 4(d), we may measure the mobility $F$. As seen in Fig. 5(d), our numerical data are in excellent agreement with the expression $F_{S}$ given in formula (2), even if 

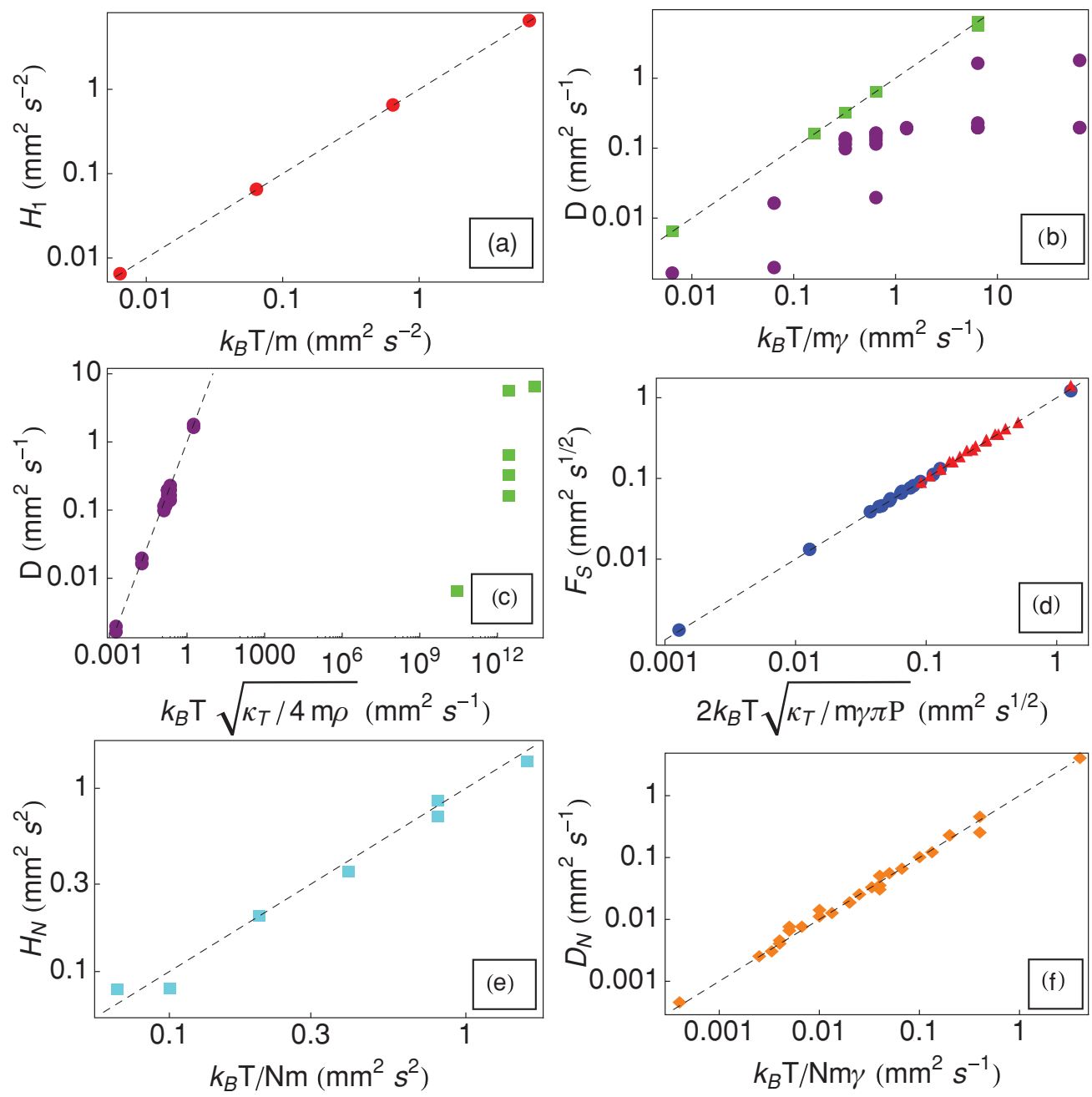

FIG. 5. (Color online) All axes are in logarithmic scales. All dotted lines are of slope 1. (a) Coefficient $H_{1}$ as a function of $k_{B} T / m$ (both in $\left.\mathrm{mm}^{2} \mathrm{~s}^{-2}\right)$. (b) Coefficient of diffusion $D$ according to $k_{B} T /(m \gamma)$ [both in $\mathrm{mm}^{2} \mathrm{~s}^{-1}$; see Eq. (15)]. The green squares represent $D$ for systems of low densities $\left(\rho \approx 100\right.$ and $\left.33 \mathrm{~m}^{-1}\right)$ and the purple circles represent $D$ for systems with higher densities $\left(\rho \approx 533 \mathrm{~m}^{-1}\right)$. (c) Coefficient of diffusion $D$ according to $k_{B} T \sqrt{\kappa_{T}} /(2 \sqrt{m \rho})$ [both in $\mathrm{mm}^{2} \mathrm{~s}^{-1}$; the numerical coefficient is slightly different from that of Eq. (16)]. The green squares represent $D$ for systems of low densities $\left(\rho \approx 100\right.$ and $\left.33 \mathrm{~m}^{-1}\right)$ and the purple circles represent $D$ for systems with higher densities $\left(\rho \approx 533 \mathrm{~m}^{-1}\right)$. (d) Mobility $F_{S}$ according to $2 k_{B} T \sqrt{\kappa_{T}} / \sqrt{\pi m \gamma \rho}$ [both in $\mathrm{mm}^{2} \mathrm{~s}^{1 / 2}$; see Eq. (2)]. The blue disks correspond to overdamped systems and the red triangles to underdamped systems. (e) Coefficient $H_{N}$ according to $k_{B} T /(N m)$ [both in $\mathrm{mm}^{2} \mathrm{~s}^{-2}$; see Eq. (17)]. (f) Coefficient of diffusion $D_{N}$ according to $k_{B} T /(N \gamma)$ [both in $\mathrm{mm}^{2} \mathrm{~s}^{-1}$; see Eq. (18)].

our system is underdamped. We discuss in Sec. IV D the case of finite systems.

The numerical data displayed in Figs. 4(c) and Fig. 4(d) are calculated for parameter values that are very close (in particular, the system sizes are equal) to the relevant parameters of the experiments reported in Ref. [25]. One can check (see Fig. 5 of Ref. [25]) that the value of the MSD is the same. The duration of the experiments is insufficient to see the final $D_{N} t$ scaling described in the next section.

\section{The long-time regime (regime III)}

It is quite intuitive that, for very long times and finite systems, all particles become fully correlated and behave as a single effective particle of mass $\mathrm{Nm}$. It is a property of the translationally invariant mode (see Sec. IV A). For small values of $\gamma$ and $N$, the MSD grows according to $H_{N} t^{2}$, with a prefactor $H_{N}$ that is different from the constant $H_{1}$ introduced in Sec. III A. $H_{N}$ should thus be given by the resolution of the Langevin equation for a free particle of mass $\mathrm{Nm}$ :

$$
H_{N}=\frac{k_{B} T}{N m},
$$

which is in very good agreement with our simulations as shown by Fig. 5(e).

At higher values of $\gamma$, and for larger systems, we only observe a linear evolution of the $\operatorname{MSD}\left\langle\Delta x^{2}\right\rangle=D_{N} t$ as shown by Figs. 4(c) and 4(d). Similarly, $D_{N}$ should be given by the 

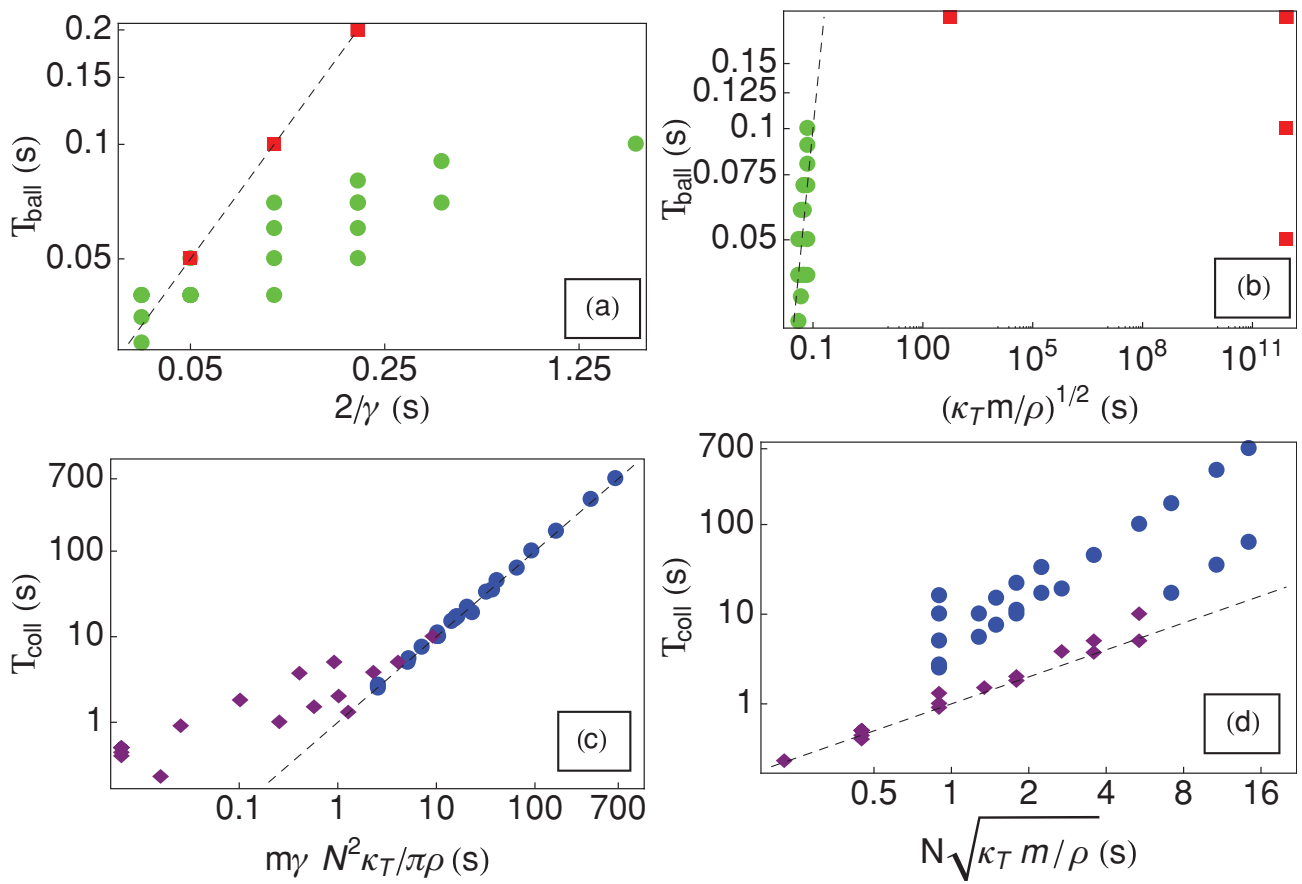

FIG. 6. (Color online) All axes are in seconds. All dashed lines are of slope 1. (a) Measures of the transition time $\tau_{\text {ball }}$ according to $2 / \gamma$ [log scale; see Eq. (19)]. The green circles represent $\tau_{\text {ball }}$ for systems of high densities $\left(\rho \approx 533 \mathrm{~m}^{-1}\right)$ and the red squares represent $\tau_{\text {ball }}$ for systems with lower densities $\left(\rho \approx 100\right.$ and $\left.33 \mathrm{~m}^{-1}\right)$. (b) Measures of the transition time $\tau_{\text {ball }}$ according to $\sqrt{\kappa_{T} m / \rho}[\log$ scale; see Eq. (20)]. The green circles represent $\tau_{\text {ball }}$ for systems of high densities $\left(\rho \approx 533 \mathrm{~m}^{-1}\right)$ and the red squares represent $\tau_{\text {ball }}$ for systems with lower densities $\left(\rho \approx 100\right.$ and $33 \mathrm{~m}^{-1}$ ). (c) Measures of the transition time $\tau_{\text {coll }}$ according to $m \gamma N^{2} \kappa_{T} / \pi \rho$ [log scale; see Eq. (21)]. The blue circles represent $\tau_{\text {coll }}$ for systems with $(N / 2 \pi) \sqrt{\gamma^{2} m \kappa_{T} / \rho}>1$ and the purple diamonds represent $\tau_{\text {coll }}$ for systems with $(N / 2 \pi) \sqrt{\gamma^{2} m \kappa_{T} / \rho}<1$ [see Eq. (40)]. (d) Measures of the transition time $\tau_{\text {coll }}$ according to $N \sqrt{\kappa_{T} m / \rho}$ [log scale; the numerical coefficient is slightly different from that of Eq. (23)]. The blue circles represent $\tau_{\text {coll }}$ for systems with $(N / 2 \pi) \sqrt{\gamma^{2} m \kappa_{T} / \rho}>1$ and the purple diamonds represent $\tau_{\text {coll }}$ for systems with $(N / 2 \pi) \sqrt{\gamma^{2} m \kappa_{T} / \rho}<1$ [see Eq. (40)].

resolution of the Langevin equation for a free particle of mass $\mathrm{Nm}$ :

$$
D_{N}=\frac{k_{B} T}{N m \gamma}
$$

This expression is in very good agreement with our results in Fig. 5(f). The Fickian behavior at very long times has already been observed in numerical simulations of SFD in finite length channels [37] and is also suggested in Ref. [38].

The interpretation of Sec. IV C shows that this regime is dominated by the collective behavior of the particles, so that we call $\tau_{\text {coll }}$ the time at which these collective behaviors takes place. We recover the values of $D_{N}$ and $H_{N}$ in Sec. IV C from our analytical solution (34) and give an estimate showing that the quadratic scaling is favored by small damping constants and small particle number, as is the case in Figs. 4(a) and 4(b).

\section{Crossover times}

Now that we know the evolution of $\left\langle\Delta x(t)^{2}\right\rangle$ in the different regimes, we can estimate the different crossover times. In this section, we proceed heuristically, defining the crossover times by requiring continuity of the MSD for successive scalings.

Following this method, the ballistic time $\tau_{\text {ball }}$ will be the time for which the curves of equations $H_{1} t^{2}$ and $2 D t$ will intersect. Depending on the expression of the diffusion coefficient $D$, we obtain

$$
\frac{k_{B} T}{m} \tau_{\text {ball }}^{2} \sim 2 \frac{k_{B} T}{m \gamma} \tau_{\text {ball }} \quad \Longrightarrow \quad \tau_{\text {ball }} \sim \frac{2}{\gamma}
$$

for weakly interacting systems. For strongly interacting systems, one has to consider the effective diffusion coefficient $D$ of Eq. (16), which gives

$$
\frac{k_{B} T}{m} \tau_{\text {ball }}^{2} \sim \frac{k_{B} T}{\pi} \sqrt{\frac{\kappa_{T}}{m \rho}} \tau_{\text {ball }} \Longrightarrow \tau_{\text {ball }} \sim \frac{1}{\pi} \sqrt{\frac{\kappa_{T} m}{\rho}} .
$$

We performed measurements of $\tau_{\text {ball }}$ for different values of parameters and report them in Figs. 6(a) and 6(b). One can clearly see that two different mechanisms are at stake: the green circles that represent systems of high densities ( $\rho \approx 533 \mathrm{~m}^{-1}$ ), which are associated with strongly interacting particles, can be easily distinguished from the red squares that represent systems with lower densities $\left(\rho \approx 100\right.$ and $33 \mathrm{~m}^{-1}$ ), thus weakly interacting particles. Formula (19) seems in good agreement with the transition times of weakly interacting systems, whereas formula (20) fits the values of $\tau_{\text {ball }}$ for strongly interacting ones. 
Let us now consider $\tau_{\text {coll }}$. For overdamped and large systems, it will be the time for which the curves of equation $F_{S} \sqrt{t}$ and $D_{N} t$ intersect, thus giving

$$
\begin{aligned}
F_{S} \sqrt{\tau_{\text {coll }}}=2 D_{N} \tau_{\text {coll }} & \Longrightarrow \tau_{\text {coll }}=\frac{F^{2}}{4 D_{N}^{2}}=\frac{S(0,0)^{2}}{D_{0}^{2}} D_{\text {eff }} \frac{N^{2}}{\pi \rho^{2}} \\
& =\frac{m \gamma N^{2} \kappa_{T}}{\pi \rho} .
\end{aligned}
$$

Note that starting from Eq. (2), using the fact that $D_{\text {eff }}=$ $D_{0} / S(0,0)$, and introducing the length $L=N / \rho$ of the chain, we may recast this expression to obtain

$$
\tau_{\mathrm{coll}}=\frac{L^{2}}{\pi D_{\mathrm{eff}}}
$$

which is interesting as it tells us that $\tau_{\text {coll }}$ can be seen as the time necessary for a given particle to diffuse over the length of the system $L$, with the effective diffusion coefficient that takes into account its interactions with the other particles.

In the case of small damping and small systems, the SFD behavior is not observed in the intermediate regime [see Figs. 4(a) and 4(b)], being replaced by a $D t$ scaling with $D$ given by Eq. (16), and the collective regime begins by a $t^{2}$ evolution [see Sec. III C and Eq. (17)]. The time $\tau_{\text {coll }}$ may thus be estimated by

$$
\begin{aligned}
2 D \tau_{\text {coll }}=H_{N} \tau_{\text {coll }}^{2} & \Longrightarrow \frac{k_{B} T}{\pi \sqrt{m K}} \tau_{\text {coll }}=\frac{k_{B} T}{N m} \tau_{\text {coll }}^{2} \\
& \Longrightarrow \tau_{\text {coll }}=\frac{N}{\pi} \sqrt{\frac{m}{K}} .
\end{aligned}
$$

One can see in Fig. 6(c) that $\tau_{\text {coll }}$ is in very good agreement with Eq. (21) for large values of $\gamma N^{2}$. For small values of $\gamma N^{2}$, the data rather follow Eq. (23), according to the analysis provided in Sec. IV C, particularly Eq. (38).

\section{THEORETICAL ANALYSIS}

\section{A. A chain of springs and point masses in a thermal bath}

In order to analyze the results presented in Sec. III, we have studied the Langevin dynamics of a chain of $N$ beads of mass $m$, aligned along the $x$ axis, interacting with a pair potential $U(x)$, with nearest neighbor interactions. Those two simplifying assumptions are, as we will see, in excellent agreement with the actual dynamics.

Small oscillations around the equilibrium position are described by linear springs of force constant $K=U^{\prime \prime}(1 / \rho)$, where $\rho$ is the particle density at equilibrium [39]. Let $x(l, t)$ be the position of particle $l$ at time $t$. The equation of motion reads

$$
\begin{aligned}
\frac{d^{2}}{d t^{2}} x(l, t)= & -\gamma \frac{d}{d t} x(l, t)+\frac{K}{m}[x(l+1, t)-2 x(l, t) \\
& +x(l-1, t)]+\frac{\mu(l, t)}{m},
\end{aligned}
$$

with the same notations as in Sec. II A. Let us consider a chain with periodic boundary conditions. We may introduce the discrete Fourier transform

$$
X(q, t)=\sum_{l=1}^{N} e^{i q l} x(l, t), \quad x(l, t)=\frac{1}{N} \sum_{k=1}^{N} e^{-i q_{k} l} X\left(q_{k}, t\right),
$$

with $q_{k}=-\pi+2 \pi k / N$ for $k=1, \ldots, N$. From now on, we simplify the notations, dropping the dependency of the modes $q_{k}$ on the natural number $k$ and replacing summations on $k$ by summations on $q$. The variance of the displacement $x$ may be calculated from the Fourier modes $X(q, t)$ as $\left\langle\Delta x^{2}(t)\right\rangle=$ $\sum_{q}\left\langle\Delta X^{2}(q, t)\right\rangle / N^{2}$, with

$$
\begin{aligned}
\left\langle\Delta X^{2}(q, t)\right\rangle \equiv & \langle[X(q, t)-\langle X(q, t)\rangle][X(-q, t) \\
& -\langle X(-q, t)\rangle]\rangle .
\end{aligned}
$$

Let us first consider the mode $q=0$, which will be noted simply $X(t)$. It follows that

$$
\frac{d^{2}}{d t^{2}} X(t)+\gamma \frac{d}{d t} X(t)=\frac{\mu(q=0, t)}{m} .
$$

Physically, this is the equation for a free particle of mass $m$ in a thermal bath at temperature $T$, with damping constant $\gamma$. The solution is composed of two parts: $X_{d}(t)$, which corresponds to the deterministic motion of the particle, and the fluctuating part $X_{\mu}(t)$, which depends linearly on the random forcing $\mu(q=0, t)$. It reads

$$
\begin{aligned}
X(t)-X^{0}= & \frac{\dot{X}^{0}}{\gamma}\left[1-e^{-\gamma t}\right]+\frac{1}{m} \int_{0}^{t} d t^{\prime} \\
& \times \int_{0}^{t^{\prime}} d t^{\prime \prime} e^{-\gamma\left(t^{\prime}-t^{\prime \prime}\right)} \mu\left(q=0, t^{\prime \prime}\right),
\end{aligned}
$$

where $X^{0} \equiv X(t=0)$ and $\dot{X}^{0} \equiv \dot{X}(t=0)$ are the initial conditions. The corresponding contribution to the MSD measured in the simulations is the double average defined by Eq. (14). It is calculated in Appendix A and reads

$$
\left\langle\Delta X^{2}\right\rangle=2 \frac{N k_{B} T}{m \gamma}\left[t-\frac{1}{\gamma}\left(1-e^{-\gamma t}\right)\right] .
$$

The translationally invariant mode $q=0$ scales as $t^{2}$ at small time $t \leqslant 1 / \gamma$ and then scales as $t$ when $t \gg 1 / \gamma$.

We now consider the modes $q \neq 0$. Using the periodic boundary conditions $x(l, t)=x(l+N, t)$, we see that each mode $X(q, t)$ of wave number $q \neq 0$ follows the equation

$\frac{d^{2}}{d t^{2}} X(q, t)+\gamma \frac{d}{d t} X(q, t)+\frac{2 K}{m}(1-\cos q) X(q, t)=\frac{\mu(q, t)}{m}$.

The roots of the characteristic polynomial associated with this equation are

$$
\omega_{ \pm}(q) \equiv-\frac{\gamma}{2} \pm \sqrt{\frac{\gamma^{2}}{4}-\omega_{q}^{2}}, \quad \omega_{q}^{2} \equiv 2 \frac{K}{m}(1-\cos q) .
$$

Physically, the mode $X(q, t)$ behaves as a particle of mass $m$ in a harmonic potential well with pulsation $\omega_{q}$, forced by 


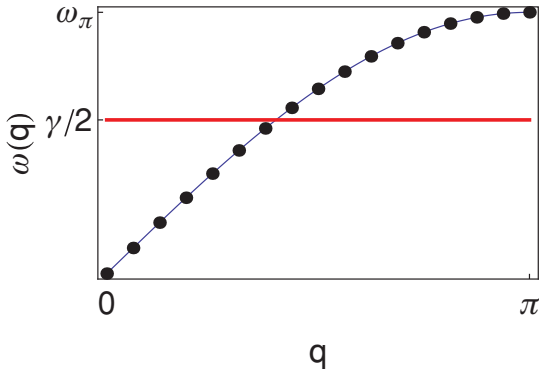

FIG. 7. (Color online) Dispersion relation $\omega(q)$ as a function of $q$, for $q \geqslant 0$ (the curve is obviously symmetric for $q \leqslant 0$ ). The continuous line is valid for the infinite chain; the dots represent the modes for $N=32$. The modes such that their frequency is less than $\gamma / 2$ are overdamped; the other ones are underdamped.

the random force $\mu(q, t)$. The solution of Eq. (30) is readily obtained as

$$
\begin{aligned}
X(q, t)= & \frac{\dot{X}(q, 0)+\omega_{-}(q) X(q, 0)}{\omega_{+}(q)-\omega_{-}(q)} e^{\omega_{+}(q) t} \\
& +\frac{\omega_{+}(q) X(q, 0)-\dot{X}(q, 0)}{\omega_{+}(q)-\omega_{-}(q)} e^{\omega_{-}(q) t}+X_{\mu}(q, t) .
\end{aligned}
$$

The modes with nonzero wave number scale as $k_{B} T t^{2} /(\mathrm{Nm})$ at small times and saturate toward the constant value $2 k_{B} T /\left(N m \omega_{q}^{2}\right)$ at very long times.

At intermediate time, the behavior of the modes $q \neq 0$ is determined by the relative values of $\omega_{q}$ and $\gamma / 2$, as illustrated in Fig. 7.

(i) The modes such that $\omega_{q}<\gamma / 2$ are overdamped. They reach their saturation value at the time $t_{\text {sat }} \sim 1 /\left|\omega_{+}(q)\right|$. The shortest saturation time is associated with $q=\pi$ and reads $t_{\text {sat }}^{\min } \sim \gamma / \omega_{\pi}^{2}$ if $\omega_{\pi}<\gamma / 2$ or $t_{\text {sat }}^{\min } \sim 2 / \gamma$ otherwise.

(ii) The modes such that $\omega_{q}>\gamma / 2$ are underdamped. They oscillate at the frequency $\omega(q) \equiv \sqrt{\omega_{q}^{2}-\gamma^{2} / 4}$. Below a time which is roughly $1 / \omega(\pi)$, all underdamped modes scale as $t^{2}$.

We do not distinguish the overdamped $\left(\gamma>2 \omega_{q}\right)$ and the underdamped $\left(\gamma<2 \omega_{q}\right)$ modes, because the final result (33) is

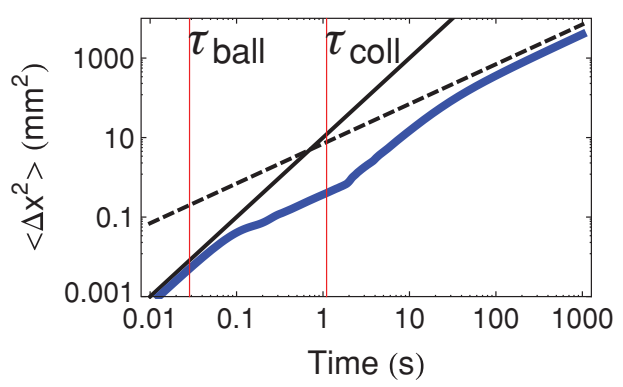

FIG. 8. (Color online) Plot of $\left\langle\Delta x(t)^{2}\right\rangle$ (in $\mathrm{mm}^{2}$ ) according to the time (in s), in logarithmic scale, calculated from the analytical solution (34) for a density $\rho=533 \mathrm{~m}^{-1}, N=32, T=10^{12} \mathrm{~K}, \Gamma \approx 6.8$, and $\gamma=0.1 \mathrm{~s}^{-1}$. The solid line is of slope 2 and the dashed line is of slope 1. This plot is to be compared to the relevant plot in Fig. 4(a). We have indicated the times $\tau_{\text {ball }}$ and $\tau_{\text {coll }}$, respectively, given by Eqs. (36) and (38). We see that both equations provide lower bounds for $\tau_{\text {coll }}$. in both cases a real function with the same formal expression. The averaging process is explained in Appendix A. One gets

$$
\begin{aligned}
\left\langle\Delta X^{2}(q, t)\right\rangle= & \frac{2 N k_{B} T}{m \omega_{q}^{2}}\left[1+\frac{\omega_{-}(q) e^{\omega_{+}(q) t}}{\omega_{+}(q)-\omega_{-}(q)}\right. \\
& \left.-\frac{\omega_{+}(q) e^{\omega_{-}(q) t}}{\omega_{+}(q)-\omega_{-}(q)}\right] .
\end{aligned}
$$

We remark that the limit $q \rightarrow 0$ is not singular, and by taking it properly in this expression one recovers Eq. (29). We think that it is nevertheless physically convenient to distinguish between the translationally invariant mode $q=0$ and the others, because they lead to different asymptotic behaviors.

Using Eq. (33) together with Eq. (29), we obtain the MSD as

$$
\begin{aligned}
\left\langle\Delta x^{2}(t)\right\rangle= & \frac{2 k_{B} T}{N m}\left\{\frac{t}{\gamma}-\frac{1}{\gamma^{2}}\left(1-e^{-\gamma t}\right)\right. \\
& \left.+\sum_{q \neq 0} \frac{1}{\omega_{q}^{2}}\left[1+\frac{\omega_{-} e^{\omega_{+} t}}{\omega_{+}-\omega_{-}}-\frac{\omega_{+} e^{\omega_{-} t}}{\omega_{+}-\omega_{-}}\right]\right\},
\end{aligned}
$$

which is the basis for the following discussion.

In the limit of very large damping, that is in the absence of the inertial term, a solution to Eq. (24) has been provided by Sjögren [24]. We have thus extended his calculations to underdamped systems ${ }^{1}$ and calculated the MSD in a different way to take into account our peculiar way of averaging (14). We also extend this discussion, in the rest of this section, to the case of finite systems.

\section{B. The ballistic regime (regime I)}

Since the small-time behavior of each mode in Eq. (34) is $\left(k_{B} T / N m\right) t^{2}$, and there are $N$ equivalent contributions to the sum, we get

$$
\left\langle\Delta x^{2}(t)\right\rangle \stackrel{t \rightarrow 0}{\sim} \frac{k_{B} T}{m} t^{2} .
$$

This result is independent of $N$ and thus valid in the thermodynamic limit too. Because of the inertial term in the Langevin equation (24), at very small times each particle behaves independently from the others and undergoes ballistic flight at the thermal velocity $\sqrt{k_{B} T / m}$.

In order to discuss the duration $\tau_{\text {ball }}$ of this regime, let us assume a finite, but large (in a sense to be defined later), particle number $N$. The time evolution of $\left\langle\Delta x^{2}(t)\right\rangle$ is determined by the mode $q=0$ and a summation on all modes $q \neq 0$. All modes in the summation (34), hence the sum itself, behave as $t^{2}$ on a time scale such that

$$
t \leqslant \tau_{\text {ball }} \equiv \min \left(\frac{2}{\gamma}, \frac{1}{\sqrt{\omega_{\pi}^{2}-\gamma^{2} / 4}}, \frac{\gamma}{\omega_{\pi}^{2}}\right) .
$$

\footnotetext{
${ }^{1}$ More precisely $\left\langle\Delta x^{2}(t)\right\rangle$ is equal to the correlation $C(0, t)$ defined by Sjögren [24] and to $2 W(t)$ introduced by Kollmann [23]. This is easily seen from the definition (13), because with the double averaging on the initial conditions and on the random noise [see Appendix A and Eqs. (28) and (32)] we get $\left\langle\left\langle x\left(t+t_{0}\right)-x\left(t_{0}\right)\right\rangle_{e}\right\rangle_{0}=0$, and then stationarity ensures $\left\langle\left\langle\left[x\left(t+t_{0}\right)-x\left(t_{0}\right)\right]^{2}\right\rangle\right\rangle=\left\langle\left\langle[x(t)-x(0)]^{2}\right\rangle\right\rangle$, which is precisely the definition of Sjögren and Kollmann.
} 
For weakly interacting or equivalently low density systems $\tau_{\text {ball }} \approx 2 / \gamma$, which was already heuristically derived in Eq. (19) and is shown in Fig. 6(a). For strongly interacting or equivalently high density systems, we get $\tau_{\text {ball }} \approx 1 / \omega_{\pi} \propto$ $\sqrt{m / K} \propto \sqrt{m \kappa_{T} / \rho}$, in perfect agreement with our observations [Fig. 6(b)] and the heuristic derivations (16) and (20).

\section{The collective regime (regime III)}

This collective regime (regime III of Fig. 3, see Sec. III C) is a property of finite systems only. This asymptotic behavior may be easily deduced from the sum (34), which is dominated by the contribution of the mode $q=0$ that scales as $\left[2 k_{B} T /(N m \gamma)\right] t$. This corresponds to the free diffusion of a particle of mass $\mathrm{Nm}$ in a thermal bath at temperature $T$. At very long times, the particles are completely correlated and behave as a single particle of effective mass, the sum of all masses. The same result has been obtained in Ref. [20].

It is not difficult to estimate the time $\tau_{\text {coll }}$. It is the time at which the contribution of the mode $q=0$ dominates the sum of the contributions of all $N-1$ other modes. Let us use the simplifying Debye approximation $\omega_{q}^{2}=(K / m) q^{2}$. We thus get

$$
\begin{aligned}
\frac{2 k_{B} T}{N m \gamma} \tau_{\text {coll }} & \sim \sum_{q \neq 0} \frac{2 k_{B} T}{N m \omega_{q}^{2}} \sim \frac{2 N k_{B} T}{4 \pi^{2} K} \sum_{i=1}^{(N-1) / 2} \frac{1}{i^{2}} \\
\Longrightarrow \tau_{\text {coll }} & \sim \frac{N^{2} m \gamma}{12 K},
\end{aligned}
$$

where we have used the fact that the sum is the generalized harmonic number $H_{(N-1) / 2,2}$ which is equal to $\pi^{2} / 6$ up to corrections of order $1 / N$. The fact that this time scales as $N^{2}$ explains why this long time regime is seldom seen in simulations (see, however, Refs. [33] and [36]) or experiments. This is clearly illustrated by our Fig. 4(b), where we show that increasing $N$ shifts the long time regime toward longer times. This expression of $\tau_{\text {coll }}$ is equal to our previous heuristic estimate (21). It means that the reasoning at the basis of the derivation of Eq. (37) includes in the right way the physical origin of the long-time collective behavior of the finite chain.

As was already quoted in Sec. III C, at very small damping constant it is possible to observe at long times the evolution $\left\langle\Delta x^{2}(t)\right\rangle=H_{N} t^{2}$. This is possible if the modes $q \neq 0$ are saturated while the mode $q=0$ still evolves as $\left[k_{B} T /(N m)\right] t^{2}$. This requires $t<1 / \gamma$ (otherwise the mode $q=0$ scales as $t$ ) and that the contribution of the $q \neq 0$ modes to the sum in Eq. (34) be less than that of the mode $q=0$. Roughly speaking, we get

$$
\begin{aligned}
\left.\left\langle\Delta x^{2}\left(t=\frac{1}{\gamma}\right)\right\rangle\right|_{q=0} \sim \frac{k_{B} T}{N m}\left(\frac{1}{\gamma}\right)^{2}>\sum_{q \neq 0} \frac{2 k_{B} T}{N m \omega_{q}^{2}} \sim \frac{N m k_{B} T}{12 K} \\
\Longrightarrow \gamma^{2}<\frac{12 K}{N^{2} m},
\end{aligned}
$$

where we have used the Debye approximation to estimate the contribution of the $q \neq 0$ modes. This means that this regime is to be observed at small damping $\gamma$ and small particle number
$N$, which is precisely the case in Figs. 4(a) and 4(b). In this case, the estimate (37) should be replaced by

$$
\begin{aligned}
& \frac{k_{B} T}{N m} \tau_{\text {coll }}^{2} \sim \sum_{q \neq 0} \frac{2 k_{B} T}{N m \omega_{q}^{2}} \sim \frac{2 N k_{B} T}{4 \pi^{2} K} \sum_{i=1}^{(N-1) / 2} \frac{1}{i^{2}} \\
& \Longrightarrow \tau_{\text {coll }} \sim N \sqrt{\frac{m}{6 K}} .
\end{aligned}
$$

This expression of $\tau_{\text {coll }}$ is equal to our previous heuristic estimate (23), allowing us to interpret the $t^{2}$ scaling at long time, in systems of few particles with small damping, as a collective behavior linked to the translationally invariant mode. This regime takes place when the greatest saturation time of the mode $q \neq 0$ is smaller than the time above which the mode $q=0$ evolves as $t$ rather than $t^{2}$. The behavior $\left\langle\Delta x^{2}(t)\right\rangle=H_{N} t^{2}$ may thus be observable when

$$
\frac{1}{\omega_{2 \pi / N}}<\frac{1}{\gamma} \Longrightarrow \frac{N}{2 \pi} \sqrt{\frac{\gamma^{2} m \kappa_{T}}{\rho}}<1 .
$$

The relevance of this estimate is proved by Figs. 6(c) and 6(d). It also shows that the time $\tau_{\text {lin }}$ introduced in Sec. IV is equal to $1 / \gamma$.

\section{The correlated regime (regime II)}

Let us now discuss the intermediate regime ${ }^{2}$ which, between the individual ballistic regime (regime I) and the collective behavior (regime III), exhibits the correlated behavior of the particles. While at asymptotically long times, all modes with finite (nonzero) wave numbers have reached a constant value, in the intermediate regime, the physical behavior of the chain at a given time $t$ results from a subtle balance between the modes that are already saturated and those that still evolve. To simplify somewhat the discussion, we consider the limit $\gamma \ll 2 \omega_{2 \pi / N}$ when all modes are oscillating (underdamped) and the limit $\gamma \gg 2 \omega_{\pi}$ when all modes are overdamped.

Let us first assume a very low damping. The modes oscillate until they reach a stationary value. The time evolution of $\left\langle\Delta x^{2}(t)\right\rangle$ is due to the progressive disappearance of the contributions of the first oscillation of those modes $q \neq 0$ that have reached their maximum value. In Fig. 9(a) this is graphically illustrated with several underdamped modes $\left(\gamma / 2=0.05 \mathrm{~s}^{-1} \ll \omega_{\pi}=25 \mathrm{~s}^{-1}\right)$, together with the complete sum (34). As a first approximation,

$$
\frac{1}{N^{2}}\left\langle\Delta X^{2}(q, t)\right\rangle \sim \frac{2 k_{B} T}{N m \omega_{q}^{2}}\left[1-e^{-\gamma t / 2} \cos \omega(q) t\right] \sim \frac{k_{B} T}{N m} t^{2} .
$$

At a given time $t$, the sum is dominated by the contributions of the modes that have not reached their first maximum, that is, those modes such that $t<1 / \omega_{q}$. Let $n(t)$ be the number of such modes. In the Debye approximation $\omega_{q}=q \sqrt{K / m}$, the maximum wave number of those modes is $1 / t \sqrt{K / m}$ so that an estimate of $n(t)$ is given by $n(t) \sim 2(N / 2 \pi)(1 / t \sqrt{K / m})$

\footnotetext{
${ }^{2}$ In simulations of the overdamped Langevin equation, for which the ballistic regime cannot be seen, this intermediate regime is the first to be observed $[15,27,28,34]$.
} 

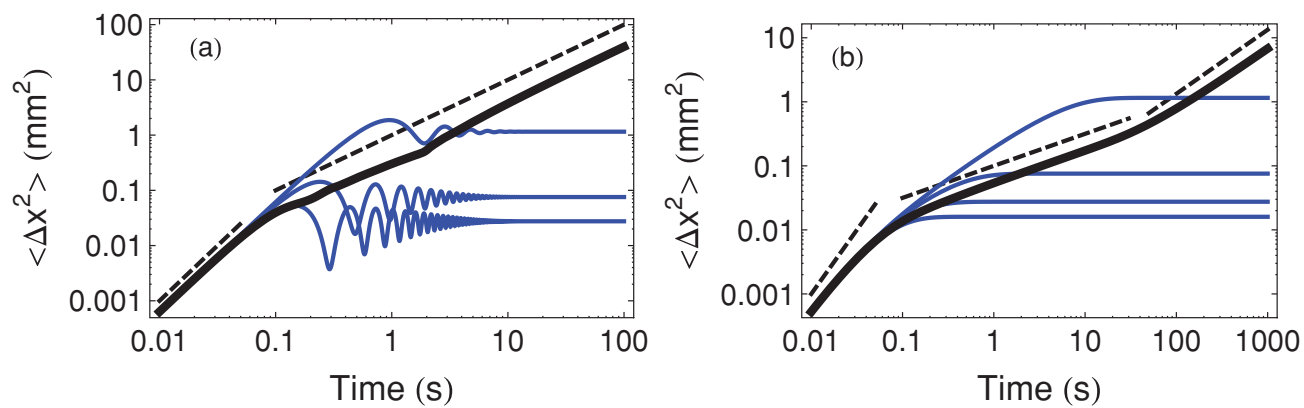

FIG. 9. (Color online) Thick solid line: Plot of $\left\langle\Delta x(t)^{2}\right\rangle$ (in $\mathrm{mm}^{2}$ ) according to the time (in s), in logarithmic scale, for a density $\rho=533 \mathrm{~m}^{-1}$, $N=32, T=10^{12} \mathrm{~K}$, and $\Gamma \approx 6.8$, calculated from (34) for the relevant damping constant. (a) Underdamped regime, $\gamma=1 \mathrm{~s}^{-1}$. The blue (gray) curves are, from top to bottom, the modes $q=\pi / 16, q=\pi / 4$, and $q=7 \pi / 16$. The dashed lines are, from left to right, of slopes 2 and 1. (b) Overdamped regime, $\gamma=60 \mathrm{~s}^{-1}$. The blue (gray) curves are, from top to bottom, the modes $q=\pi / 16, q=\pi / 4, q=7 \pi / 16$, and $q=5 \pi / 8$. The dashed lines are, from left to right, of slopes $2,1 / 2$, and 1 .

(the factor 2 takes into account the modes $\pm|q|$ ). The variance may thus be estimated as

$$
\left\langle\Delta x^{2}(t)\right\rangle \sim \frac{k_{B} T}{N m} t^{2} n(t) \sim \frac{k_{B} T}{\pi \sqrt{m K}} t .
$$

This is a normal diffusion with a diffusivity $k_{B} T /(2 \pi \sqrt{m K})$ that depends on the stiffness of the interaction $K$, showing that it is a collective effect. This is in agreement with our observations, see Fig. 5(c).

In the opposite limit of a very strong damping, all modes evolve monotonously toward their saturation value. As a first approximation,

$$
\begin{aligned}
\frac{1}{N^{2}}\left\langle\Delta X^{2}(q, t)\right\rangle & \sim \frac{2 k_{B} T}{N m \omega_{q}^{2}}\left\{1+\frac{\omega_{-}(q)\left[1-\omega_{+}(q) t\right]}{\omega_{+}(q)-\omega_{-}(q)}\right\} \\
& \sim \frac{2 k_{B} T}{N m \gamma} t,
\end{aligned}
$$

where we have used $\omega_{+}(q)-\omega_{-}(q) \approx \gamma$ and $\omega_{+}(q) \approx \omega_{q}^{2} / \gamma$ and $\omega_{-}(q) \approx-\gamma$. A mode is saturated at a time $t>\gamma / \omega_{q}^{2}$. At a given time $t$, in the Debye approximation, the modes that increase with time are such that $q<\sqrt{m \gamma /(K t)}$. This reasoning is graphically illustrated in Fig. 9(a) where we show several overdamped modes $\left(\gamma / 2=30 \mathrm{~s}^{-1}>\omega_{\pi}=\right.$ $\left.25 \mathrm{~s}^{-1}\right)$, together with the complete sum (34). Their number is thus $n(t) \sim 2(N / 2 \pi) \sqrt{m \gamma /(K t)}$. The contributions of all such modes thus give

$$
\begin{aligned}
\left\langle\Delta x^{2}(t)\right\rangle & \sim \frac{2 k_{B} T}{N m \gamma} t n(t) \sim \frac{2 k_{B} T}{N m \gamma} t \frac{2 N}{2 \pi} \sqrt{\frac{m \gamma}{K t}} \\
& =\frac{2 k_{B} T}{\pi \sqrt{m K \gamma}} t^{1 / 2}=\frac{2 k_{B} T}{\pi} \sqrt{\frac{\kappa_{T}}{m \rho \gamma}} t^{1 / 2},
\end{aligned}
$$

where we have introduced the isothermal compressibility $\kappa_{T}$ in the last expression to ease the comparison with the exact expression of $F_{S}(2)$. Taking into account the crudeness of our approximations, this estimate is extremely satisfactory because we recover the SFD behavior $\left\langle\Delta x^{2}(t)\right\rangle \propto t^{1 / 2}$, with a prefactor that is almost the exact one.

In the general case, the modes with wave number $q$ such that $\gamma<2 \omega_{q}$ contribute to a $t$ scaling of the MSD, whereas the modes such that $\gamma>2 \omega_{q}$ contribute to the SFD behavior, that is, a $t^{1 / 2}$ scaling of the MSD. The typical time $\tau_{\text {sub }}$ at which the subdiffusive SFD behavior takes place is thus the inverse of this cutoff frequency, $\tau_{\text {sub }}=2 / \gamma$. At a given particle number $N$, the minimum nonzero frequency is $\omega_{2 \pi / N}$. If the damping constant is so small that all modes are underdamped $\left(\gamma<2 \omega_{2 \pi / N}\right)$, the MSD scales as $t$ in the collective regime II. Increasing the damping, at fixed $N$, amounts to an increase in the number of overdamped modes and favors the subdiffusive $t^{1 / 2}$ scaling for the MSD. This is exemplified by Fig. 4(a). Increasing the particle number $N$, at fixed $\gamma$, amounts to a decrease in the frequency $\omega_{2 \pi / N}$, and hence to an increase in the number of overdamped modes, and favors the subdiffusive $t^{1 / 2}$ scaling for the MSD. This is exemplified by Fig. 4(b).

The result (44) is only approximate, so that the numerical prefactor cannot be trusted, but it explains under which conditions the SFD regime may be seen in finite systems with periodic boundary conditions [we remind the reader that this latter is the key assumption leading to Eq. (30)]. In Appendix B, we prove that the expression (2) for the mobility of long-ranged interacting systems is valid in the underdamped case $\gamma<2 \omega_{\pi}$ too, in the thermodynamic limit.

\section{CONCLUSION}

In this paper, we study the SFD of a chain of particles with long-ranged interactions, without the simplifying assumption of overdamped dynamics. We have focused our discussion on finite-size effects and the influence of low damping. We use numerical simulations of the Langevin equation with the Gillespie algorithm [31,32] and model the system as a chain of linear springs (spring constant $K$ ) and point masses $(m)$ in a thermal bath at temperature $T$.

In our simulation's data, we have identified several regimes for the time evolution of the $\operatorname{MSD}\left\langle\Delta x(t)^{2}\right\rangle$. At small times $\left(0 \leqslant t \leqslant \tau_{\text {ball }}\right)$, it evolves as $\left\langle\Delta x(t)^{2}\right\rangle=\left(k_{B} T / m\right) t^{2}$. This is a ballistic flight that traces back to the inertial effects and is observed whatever the damping $\gamma$ or the particle number $N$. We recover this behavior in the thermodynamic limit $(N \rightarrow \infty$ at finite density) from our model. The prefactor of the $t^{2}$ scaling measured in our simulations is in excellent agreement with the theory.

For finite systems with periodic boundary conditions, an intermediate regime ( $\tau_{\text {ball }} \leqslant t \leqslant \tau_{\text {coll }}$ ) takes place. Depending 
on the respective values of the damping constant and the number of particles, we may observe a diffusive behavior, a SFD behavior, or successively both. We provide a physical explanation of those observations when we express the motion of the chain in terms of normal modes of oscillations. The MSD of the chain results from the superposition of all those modes. The mode associated with the null wave number is always overdamped, and is similar to the motion of a free particle in a thermal bath, since no restoring force is exerted on it. The modes of finite (nonzero) wave numbers have the same dynamics as an oscillator in a harmonic well. At long time, the MSD of all modes with nonzero wave numbers saturates toward a constant value. The overdamped modes, which do not oscillate until they saturate, contribute to the SFD scaling $\left\langle\Delta x(t)^{2}\right\rangle \propto t^{1 / 2}$. The underdamped modes oscillate before their saturation and contribute to the linear scaling $\left\langle\Delta x(t)^{2}\right\rangle \propto t$.

In the thermodynamic limit, for systems of infinite number of particles, we exhibit analytically the SFD behavior $\left\langle\Delta x(t)^{2}\right\rangle=F_{S} t^{1 / 2}$ at asymptotically long times. We recover the mobility $F_{S}$ that was previously calculated for long-ranged interactions and overdamped dynamics [23,24], thus extending the previous calculation to systems with arbitrary damping.

At asymptotically long times $\left(t \gg \tau_{\text {coll }}\right)$, for a finite number of particles with periodic boundary conditions, the system behaves as an effective particle of mass $\mathrm{Nm}$. The physical origin of this behavior is the motion of the collective mode of null wave number, which is linked to the translational invariance of the system. For $t \geqslant \tau_{\text {lin }} \geqslant \tau_{\text {coll }}$, the system undergoes a linear diffusion with a diffusion coefficient $D_{N}=$ $2 k_{B} T /(N m \gamma)$. We show that this regime takes place whatever the value of $\gamma$. The duration of our simulations allows us to see this regime, and the measured diffusivity is in excellent agreement with its predicted value. We provide estimates of the time $\tau_{\text {coll }} \sim N^{2} m \gamma / K$ at large damping and $\tau_{\text {lin }} \sim 1 / \gamma$ at small damping. Those estimates are in good agreement with our simulation's data. At small particle number and small damping, a new regime takes place at times $\tau_{\text {lin }} \geqslant t \geqslant \tau_{\text {coll }}$. It corresponds to the ballistic flight of the effective particle of mass $N m$, with $\left\langle\Delta x(t)^{2}\right\rangle=\left(k_{B} T / N m\right) t^{2}$. In this case, the time $\tau_{\text {coll }} \sim N \sqrt{m / K}$.

\section{ACKNOWLEDGMENTS}

We thank J. Moukhtar and F. van Wijland for helpful discussions.

\section{APPENDIX A: AVERAGING}

In this appendix, we calculate the averages used in our analysis of the simulation's data. As explained in Sec. II B [see Eq. (14)], we perform a double averaging. The first averaging is ensemble averaging, done on the statistical distribution of the random force $\mu(t)$, and is denoted in this appendix as $\langle\cdot\rangle_{e}$ for the sake of clarity. This averaging is involved in Eqs. (4), (5), and (8). The second averaging is performed on the statistical distributions of $X^{0}$ and $\dot{X}^{0}$ and is denoted as $\langle\cdot\rangle_{0}$. It is obvious that those two averaging operations commute and that $\left\langle X_{\mu}(t)\right\rangle_{0}=X_{\mu}(t)$ and $\left\langle X_{d}(t)\right\rangle_{e}=X_{d}(t)$. In Eq. (26), the ensemble averaging is made on the random force $\mu(t)$ so that

$$
\begin{aligned}
\left\langle\Delta X^{2}(q, t)\right\rangle_{e} \equiv & \left\langle\left[X(q, t)-\langle X(q, t)\rangle_{e}\right][X(-q, t)\right. \\
& \left.\left.-\langle X(-q, t)\rangle_{e}\right]\right\rangle_{e}
\end{aligned}
$$

\section{The mode $q=0$}

For the translational invariant mode $q=0$, all trajectories beginning at a given time $t_{0}$ are equivalent, so that all initial positions $X^{0}$ are equivalent. It is easy to check that $\left\langle\left\langle X(t)-X^{0}\right\rangle_{e}\right\rangle_{0}=\left\langle X_{d}(t)\right\rangle_{0}+\left\langle X_{\mu}(t)\right\rangle_{e}=0$. For the same reason, the double average $\left\langle\left\langle X_{d}(t) X_{\mu}(t)\right\rangle_{e}\right\rangle_{0}=0$ because this term is linear in $\dot{X}^{0}$ and in $\mu(0, t)$. The variance is thus

$$
\begin{aligned}
\left\langle\left\langle\Delta X^{2}\right\rangle_{e}\right\rangle_{0}= & \left\langle\left\langle\left[X(t)-X^{0}\right]^{2}\right\rangle_{e}\right\rangle_{0}=\left\langle\left[X_{d}(t)-X^{0}\right]^{2}\right\rangle_{0} \\
& +\left\langle X_{\mu}(t)^{2}\right\rangle_{e} .
\end{aligned}
$$

The variance for the deterministic part of the displacement is

$$
\begin{aligned}
\left\langle\left\langle\Delta X_{d}^{2}(q, t)\right\rangle_{e}\right\rangle_{0} & =\frac{\left\langle\left|\dot{X}^{0}\right|^{2}\right\rangle_{0}}{\gamma^{2}}\left[1-e^{-\gamma t}\right]^{2} \\
& =\frac{N k_{B} T}{m \gamma^{2}}\left[1-e^{-\gamma t}\right]^{2},
\end{aligned}
$$

where the last expression is provided by the equipartition theorem for the potential energy.

We then have to calculate the variance for the fluctuating part, $\left\langle X_{\mu}(t)^{2}\right\rangle_{e}$. We begin by calculating the following time derivative:

$$
\begin{aligned}
\frac{d}{d t}\left\langle\Delta X_{\mu}^{2}\right\rangle_{e} & =\frac{d}{d t}\left\langle X_{\mu}(t) X_{\mu}(t)\right\rangle_{e} \\
& =\left\langle X_{\mu}(t) \dot{X}_{\mu}(t)\right\rangle_{e}+\left\langle\dot{X}_{\mu}(t) X_{\mu}(t)\right\rangle_{e} \\
& =2 \operatorname{Re}\left\langle X_{\mu}(t) \dot{X}_{\mu}(t)\right\rangle_{e} .
\end{aligned}
$$

From Eq. (28), we get

$$
\begin{aligned}
\left\langle X_{\mu}(t) \dot{X}_{\mu}(t)\right\rangle_{e}= & \frac{1}{m^{2}} \int_{0}^{t} d t^{\prime} \int_{0}^{t^{\prime}} d t^{\prime \prime} e^{-\gamma\left(t^{\prime}-t^{\prime \prime}\right)} \int_{0}^{t} d t^{\prime \prime \prime} e^{-\gamma\left(t-t^{\prime \prime \prime}\right)} \\
& \times\left\langle\mu\left(q=0, t^{\prime \prime}\right) \mu\left(q=0, t^{\prime \prime \prime}\right)\right\rangle_{e} .
\end{aligned}
$$

The correlation for the random noise is $\left\langle\mu(l, t) \mu\left(l^{\prime}, t^{\prime}\right)\right\rangle_{e}=$ $g \delta_{l l^{\prime}} \delta\left(t-t^{\prime}\right)$, with $g=2 m k_{B} T \gamma$. Besides, $\mu(q=0, t)=$ $\sum_{l=1}^{N} \mu(l, t)$; hence

$$
\begin{aligned}
\left\langle\mu(q=0, t) \mu\left(q=0, t^{\prime}\right)\right\rangle_{e} & =\sum_{l=1}^{N} \sum_{l^{\prime}=1}^{N}\left\langle\mu(l, t) \mu\left(l^{\prime}, t^{\prime}\right)\right\rangle_{e} \\
& =2 N m k_{B} T \gamma \delta\left(t-t^{\prime}\right) .
\end{aligned}
$$

Performing the integrations in Eq. (A5), we get

$$
\frac{d}{d t}\left\langle\Delta X_{\mu}^{2}\right\rangle_{e}=2 \frac{N k_{B} T}{m \gamma}\left(1+e^{-2 \gamma t}-2 e^{-\gamma t}\right),
$$

and a last integration gives the final result

$$
\left\langle\Delta X_{\mu}^{2}\right\rangle_{e}=2 \frac{N k_{B} T}{m \gamma}\left(t+\frac{1-e^{-2 \gamma t}}{2 \gamma}-2 \frac{1-e^{-\gamma t}}{\gamma}\right) .
$$

Injecting this result and Eq. (A3) in Eq. (A2) gives the final result, Eq. (29), as stated in the text. 


\section{A. 2. The modes $q \neq 0$}

Physically, the dynamics of the mode $X(q, t)$ with $q \neq 0$ is identical to the motion of a damped harmonic oscillator (30). In this case, the initial values $X(q, 0)$ correspond to an initial position in a potential well and are thus not equivalent. On the other hand, the stationary state is quickly reached and the statistical distribution on $X(q, 0)$ is readily described taking different trajectories. In the data analysis, a trajectory is given by looking at values $X\left(q, t+t_{0}\right)-X\left(q, t_{0}\right)$ and varying the time $t_{0}$ amount to varying the initial value $X(q, 0)$. The expression (26) of the variance is thus replaced by

$$
\begin{aligned}
& \left\langle\left\langle\Delta X^{2}(q, t)\right\rangle_{e}\right\rangle_{0} \\
& \equiv\left\langle\left\langle\left\langle\left[X(q, t)-X(q, 0)-\left\langle\langle X(q, t)-X(q, 0)\rangle_{e}\right\rangle_{0}\right][X(-q, t)\right.\right.\right. \\
& \left.\left.\left.\quad-X(-q, 0)-\left\langle\langle X(-q, t)-X(q, 0)\rangle_{e}\right\rangle_{0}\right]\right\rangle_{e}\right\rangle_{0},
\end{aligned}
$$

As shown by Eq. (34), $X(q, t)=X_{d}(q, t)+X_{\mu}(q, t)$, where $X_{d}(q, t)$ is the deterministic part, linear in $X(q, 0)$ and $\dot{X}(q, 0)$, and $X_{\mu}(q, t)$ is the random part. It is not necessary to give explicitly the random part, all we need to know is that it is linear in the random force $\mu(q, t)$. Since $\langle X(q, 0)\rangle_{0}=0,\langle\dot{X}(q, 0)\rangle_{0}=0$, and $\left\langle X_{\mu}(q, t)\right\rangle_{e}=0$, we have $\left\langle\langle X(q, t)-X(q, 0)\rangle_{e}\right\rangle_{0}=0$. The cumbersome expression (A9) may thus be simplified to give

$$
\begin{aligned}
&\left\langle\left\langle\Delta X^{2}(q, t)\right\rangle_{e}\right\rangle_{0} \\
&=\left\langle\langle[X(q, t)-X(q, 0)][X(-q, t)-X(-q, 0)]\rangle_{e}\right\rangle_{0} \\
&=\left\langle\langle X(q, t) X(-q, t)\rangle_{e}\right\rangle_{0}+\left\langle\langle X(q, 0) X(-q, 0)\rangle_{e}\right\rangle_{0}- \\
&-2 \operatorname{Re}\left[\left\langle\langle X(q, t) X(-q, 0)\rangle_{e}\right\rangle_{0}\right] .
\end{aligned}
$$

This may be simplified further, because the stationarity implies $\quad\left\langle\langle X(q, t) X(-q, t)\rangle_{e}\right\rangle_{0}=\left\langle\langle X(q, 0) X(-q, 0)\rangle_{e}\right\rangle_{0}=$ $N k_{B} T / K$. Moreover, since $X(-q, 0)$ does not depend on $\mu$ we have $\left\langle\langle X(q, t) X(-q, 0)\rangle_{e}\right\rangle_{0}=\left\langle X_{d}(q, t) X(-q, 0)\right\rangle_{0}$. The averaging is thus easily performed to give Eq. (33).

\section{APPENDIX B: THE CHAIN OF SPRINGS IN THE THERMODYNAMIC LIMIT}

In the thermodynamic limit $N \rightarrow \infty$, the discrete sum in Eq. (34) may be replaced by an integral (taking advantage of the fact that the expressions for $q \neq 0$ are valid in the limit $q \rightarrow 0)$

$$
\begin{aligned}
\left\langle\Delta x^{2}(t)\right\rangle= & \frac{2 k_{B} T}{m} \frac{1}{\pi} \int_{0}^{\pi} d q \frac{1}{\omega_{q}^{2}}\left[1+\frac{\omega_{-}(q) e^{\omega_{+}(q) t}}{\omega_{+}(q)-\omega_{-}(q)}\right. \\
& \left.-\frac{\omega_{+}(q) e^{\omega_{-}(q) t}}{\omega_{+}(q)-\omega_{-}(q)}\right],
\end{aligned}
$$

where we used the rule $(1 / N) \sum_{q} \longrightarrow(1 / 2 \pi) \int_{-\pi}^{\pi}$ and the invariance $q \rightarrow-q$.

In the limit $\gamma \gg 2 \omega_{\pi}$, this integral may be expressed in closed form [24]. This is not possible in the general case, but we may obtain its asymptotic behavior at long times using the Laplace method [40]. To this end, we express the time derivative

$$
\frac{\partial\left\langle\Delta x^{2}(t)\right\rangle}{\partial t}=\frac{2 k_{B} T}{m} \frac{1}{\pi} \int_{0}^{\pi} d q \frac{e^{\omega_{+}(q) t}-e^{\omega_{-}(q) t}}{\omega_{+}(q)-\omega_{-}(q)} .
$$

For underdamped modes, $\omega_{ \pm}=-\gamma / 2 \pm i \sqrt{\omega_{q}^{2}-\gamma^{2} / 4}$, the long-time behavior is dominated by $\exp [-\gamma t / 2]$. Since $\lim _{q \rightarrow 0} \omega_{q}=0$, the modes with small wave numbers are always overdamped. The asymptotic behavior of the integral is dominated by the neighborhood of the maximum of $\omega_{+}(q)$ at $q=0$, with $\omega_{+}(0)=0, \omega_{+}^{\prime}(0)=0$ and $\omega_{+}^{\prime \prime}(0)=-2 K /(m \gamma)$. The leading term in Eq. (B2) is thus [40]

$$
\begin{aligned}
& \frac{\partial\left\langle\Delta x^{2}(t)\right\rangle}{\partial t} \stackrel{t \rightarrow \infty}{\sim} \frac{2 k_{B} T}{\pi m} \frac{1}{2}\left[\frac{2}{-t \omega_{+}^{\prime \prime}(0)}\right]^{1 / 2} \frac{1}{\gamma} e^{\omega_{+}(0) t} \Gamma\left(\frac{1}{2}\right) \\
& =2\left[\frac{\left(k_{B} T\right)^{2}}{\pi m \gamma K}\right]^{1 / 2} \frac{1}{2 t^{1 / 2}} .
\end{aligned}
$$

The leading behavior of the variance, at long times, is thus

$$
\left\langle\Delta x^{2}(t)\right\rangle \stackrel{t \rightarrow \infty}{\sim} 2\left(\frac{k_{B} T D_{0} \kappa_{T}}{\pi \rho}\right)^{1 / 2} t^{1 / 2} .
$$

To get the last expression, we have used the relation $m \gamma D_{0}=$ $k_{B} T$ and introduced the isothermal compressibility of the chain, which is $\kappa_{T}=\rho / K$ [39].

We recover the behavior for SFD of particles interacting with a long-ranged potential, as was shown by Kollmann [23] (see [25] for a rewriting of Kollmann's result in terms of the isothermal compressibility) and Sjögren [24]. Those previous calculations were done under the simplifying assumption of overdamped dynamics $\left(\gamma \gg 2 \omega_{\pi}\right)$. We nevertheless recover the same result because the asymptotics of integral (B2) is dominated by the modes of small wave numbers $q \ll 1$. Whatever the finite value of the damping constant $\gamma$, they are always overdamped because $\omega_{q \rightarrow 0}=0$.
[1] A. Hodgkin and R. Keynes, J. Physiol. 128, 61 (1955).

[2] V. Gupta, S. S. Nivarthi, A. V. McCormick, and H. T. Davis, Chem. Phys. Lett. 247, 596 (1995).

[3] K. Hahn, J. Kärger, and V. Kukla, Phys. Rev. Lett. 76, 2762 (1996).

[4] T. Chou and D. Lohse, Phys. Rev. Lett. 82, 3552 (1999).
[5] P. Demontis, G. Stara, and G. Suffritti, J. Chem. Phys. 120, 9233 (2004).

[6] L. Wang, X. Gao, Z. Sun, and J. Feng, J. Chem. Phys. 130, 184709 (2009).

[7] S. Seidelin, J. Chiaverini, R. Reichle, J. J. Bollinger, D. Leibfried, J. Britton, J. H. Wesenberg, R. B. Blakestad, 
R. J. Epstein, D. B. Hume, W. M. Itano, J. D. Jost, C. Langer, R. Ozeri, N. Shiga, and D. Wineland, Phys. Rev. Lett. 96, 253003 (2006).

[8] R. Besseling, R. Niggebrugge, and P. H. Kes, Phys. Rev. Lett. 82, 3144 (1999).

[9] N. Kokubo, R. Besseling, and P. Kes, Phys. Rev. B 69, 064504 (2004).

[10] Q.-H. Wei, C. Bechinger, and P. Leiderer, Science 287, 625 (2000).

[11] B. Cui, H. Diamant, and B. Lin, Phys. Rev. Lett. 89, 188302 (2002).

[12] B. Lin, B. Cui, J.-H. Lee, and J. Yu, Europhys. Lett. 57, 724 (2002).

[13] B. Lin, M. Meron, B. Cui, S. A. Rice, and H. Diamant, Phys. Rev. Lett. 94, 216001 (2005).

[14] M. Köppl, P. Henseler, A. Erbe, P. Nielaba, and P. Leiderer, Phys. Rev. Lett. 97, 208302 (2006).

[15] P. Henseler, A. Erbe, M. Köppl, P. Leiderer, and P. Nielaba, e-print arXiv:0810.2302v1 [cond-mat].

[16] C. Lutz, M. Kollmann, P. Leiderer, and C. Bechinger, J. Phys. Condens. Matter 16, S4075 (2004).

[17] C. Lutz, M. Kollmann, and C. Bechinger, Phys. Rev. Lett. 93, 026001 (2004).

[18] T. E. Harris, J. Appl. Prob. 2,323 (1965).

[19] D. G. Levitt, Phys. Rev. A 8, 3050 (1973).

[20] H. van Beijeren, K. W. Kehr, and R. Kutner, Phys. Rev. B 28 , 5711 (1983).

[21] R. Arratia, Ann. Probab. 11, 362 (1983).

[22] P.-G. de Gennes, J. Chem. Phys. 55, 572 (1971).
[23] M. Kollmann, Phys. Rev. Lett. 90, 180602 (2003).

[24] L. Sjögren, "Stochastic Processes in Physics, Chemistry and Biology" (lecture notes), 2007.

[25] C. Coste, J.-B. Delfau, C. Even, and M. Saint Jean, Phys. Rev. E 81, 051201 (2010).

[26] G. Nägele, Phys. Rep. 272, 215 (1996).

[27] S. Herrera-Velarde and R. Castañeda Priego, J. Phys. Condens. Matter 19, 226215 (2007).

[28] S. Herrera-Velarde and R. Castañeda Priego, Phys. Rev. E 77, 041407 (2008).

[29] G. Coupier, M. Saint Jean, and C. Guthmann, Phys. Rev. E 73, 031112 (2006).

[30] G. Coupier, M. Saint Jean, and C. Guthmann, Europhys. Lett. 77, 60001 (2007)

[31] D. T. Gillespie, Phys. Rev. E 54, 2084 (1996).

[32] D. T. Gillespie, Am. J. Phys. 64, 225 (1996).

[33] K. Nelissen, V. Misko, and F. Peeters, Europhys. Lett. 80, 56004 (2007).

[34] P. M. Centres and S. Bustingorry, Phys. Rev. E 81, 061101 (2010).

[35] A. Taloni and M. A. Lomholt, Phys. Rev. E 78, 051116 (2008).

[36] D. V. Tkachenko, V. R. Misko, and F. M. Peeters, Phys. Rev. E 82, 051102 (2010).

[37] P. Nelson and S. Auerbach, J. Chem. Phys. 110, 9235 (1999).

[38] S. Vasenkov and J. Kärger, Phys. Rev. E 66, 052601 (2002).

[39] L. Brillouin, Wave Propagation in Periodic Structures (Dover, New York, 1953).

[40] A. Nayfeh, Introduction to Perturbation Techniques (Wiley, New York, 1993). 